\title{
Inappropriate ICD Discharges in Single-Chamber Versus Dual-Chamber Devices in the Pediatric and Young Adult Population
}

\author{
DAVID LAWRENCE, M.D., ${ }^{*}$ NICHOLAS VON BERGEN, M.D., $\dagger$ IAN H. LAW, M.D. $\dagger$ \\ DAVID J. BRADLEY, M.D. $\ddagger$ MACDONALD DICK II, M.D. $§$ PATRICIO A. FRIAS, M.D., ${ }^{*}$ \\ MARGARET J. STREIPER, M.D., ${ }^{*}$ and PETER S. FISCHBACH, M.D.*
}

From the *Department of Pediatrics, Sibley Heart Center-Cardiology, Emory University, Atlanta, Georgia; $\nmid$ Department of Pediatrics, University of Iowa, Iowa City, Iowa; †Department of Pediatrics, University of Utah, Salt Lake City, Utah; and $\S$ Department of Pediatrics, University of Michigan, Ann Arbor, Michigan, USA

Inappropriate ICD Discharges in Pediatric Patients. Background: Implantable cardioverter defibrillator (ICD) use is increasing in young patients and is frequently complicated by inappropriate device discharges (IDs), leading to discomfort, anxiety and, potentially, proarrhythmia. Dual-chamber (DC) ICDs may decrease IDs by improving rhythm discrimination, but are associated with increased size, expense, and implant complications. We examined whether the frequency of IDs was the same in dual- and single-chamber (SC) ICDs in young patients.

Methods: A multicenter review of patients $\leq \mathbf{3 0}$ years of age with ICDs was performed. Demographic data and number of appropriate discharges (ADs) and IDs were collected. ADs and IDs were categorized after review of the episode by the primary electrophysiologist.

Results: Of 168 subjects $(\mathrm{SC}=52$; female $=76), 41$ patients received $139 \mathrm{ADs}$, while 35 patients received 159 IDs. There were no differences related to gender or primary diagnosis, but patients with $\mathrm{SC}$ versus DC devices were younger $(12.3 \pm 5.0$ years vs $14.9 \pm 4.4$ years, $P<0.0001)$ at implant. SC and DC devices were implanted with the same frequency for primary and secondary prevention. There were no significant differences in the incidence of IDs or ADs between the patients with SC or DC devices (AD: 12/52 SC vs 29/116 DC, $P=0.79$; ID 7/52 SC vs 28/116 DC, $P=0.13$ ).

Conclusion: In this cohort of young patients, DC ICDs did not provide added protection from IDs. Hence, the added complexity and cost of an atrial lead appear unwarranted if used solely for assistance with rhythm discrimination in young patients. (J Cardiovasc Electrophysiol, Vol. 20, pp. 287-290, March 2009)

ICD, pediatric, inappropriate shock, rhythm discrimination, congenital heart disease

\section{Introduction}

Implantable cardioverter defibrillators (ICDs) have been shown to reduce mortality in several patient groups. ${ }^{1-3}$ Use of ICDs may be complicated by inappropriate device discharges (IDs). Inappropriate shocks may be due to lead dysfunction, electromagnetic noise, T-wave oversensing, or misclassification of supraventricular tachycardia (SVT). In the pediatric population, SVT (including sinus tachycardia) is a common cause of IDs. ${ }^{4,5}$ IDs cause pain, anxiety, depression, a decreased quality of life, and even death.6,7

Dual-chamber (DC) ICDs (DDD-ICD) are indicated for select patients, including those who also require antibradycardia pacing due to sinus node dysfunction, require atrial antitachycardia therapies, or have AV conduction system

Dr. Fischbach reports receiving honoraria for participation in a speakers' bureau from Boston Scientific.

Address for correspondence: Peter S. Fischbach, M.D., Department of Pediatrics, Sibley Heart Center-Cardiology, Emory University School of Medicine, 2835 Brandywine Road, Suite 300, Atlanta, GA 30341, USA. Fax: 404-785-0029; E-mail: fischbachp@kidsheart.com

Manuscript received 12 March 2008; Revised manuscript received 26 July 2008; Accepted for publication 12 August 2008.

doi: 10.1111/j.1540-8167.2008.01322.x disease. The DDD-ICD, with its ability to simultaneously analyze both atrial and ventricular activity, may offer the advantage of improved arrhythmia detection and discrimination over the single-chamber (SC) device. This is particularly important in children and young adults with ICDs who have rapid sinus rates and are more active than older patients with ICDs. This feature could potentially reduce the incidence of IDs attributable to the misclassification of sinus tachycardia or supraventricular tachycardia (SVT) as a ventricular event.

Data regarding protective benefits of DC devices compared with SC devices in adults with ICDs have been conflicting. ${ }^{8-10}$ The benefit of DDD-ICDs in reducing IDs in pediatric and young adult patients is yet to be demonstrated. As DC devices are associated with increased cost, procedure time, programming complexity, and procedure-related complications, ${ }^{11}$ it is important to try to evaluate whether there is an advantage to their use in this population. This retrospective study sought to evaluate if there was an advantage in using DDD-ICDs versus VVI-ICDs for preventing IDs in young patients.

\section{Methods}

The study was investigator initiated and managed. The pacemaker and ICD databases of four institutions (Children's Healthcare of Atlanta/Emory University, University of Michigan, University of Iowa, and the University of Utah) 


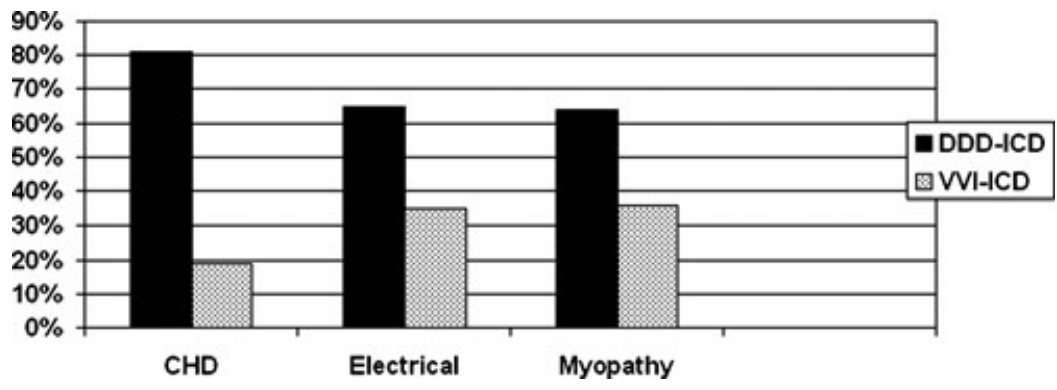

Figure 1. Distribution of ICD device type by primary diagnosis. $C H D=$ palliated congenital heart disease; Electrical = electrical disorder.

were queried for patients $\leq 30$ years of age who had an ICD implanted between October 1992 and February 2007. Patient demographics, implant indications, presence of heart disease (structural or electrical), and device therapies were recorded. The patients who experienced device activity were reviewed. The ICD diagnostic data were reviewed by the primary electrophysiologist to determine if the delivered therapy was appropriate or inappropriate. An ID was defined as a delivered ICD therapy for any reason other than detected and confirmed ventricular tachycardia (VT) or ventricular fibrillation (VF).

\section{Statistical Analysis}

The frequencies of ADs and IDs were compared using a chi-square analysis. Continuous variables were expressed as means plus or minus the standard deviation. The means were compared using Student's $t$-test analysis. A P value $<0.05$ was considered statistically significant for individual comparisons.

\section{Results}

A total of 168 patients satisfied the inclusion criteria and had at least 1 year of follow-up. Of these, $54 \%(n=92)$ were male. There were 44 patients with palliated congenital heart disease (D-transposition of the great arteries [DTGA] $\mathrm{n}=11$, tetralogy of Fallot [TOF] $\mathrm{n}=15$, other $\mathrm{n}=$ 18), 51 with cardiomyopathies (hypertrophic cardiomyopathy $[\mathrm{HCM}] \mathrm{n}=36$, arrhythmogenic right ventricular dysplasia [ARVD] $n=3$, other $n=12$ ), and 72 with primary electrical disease (long QT syndrome [LQTS] n $=49$, catecholaminergic polymorphic ventricular tachycardia [CPVT] $n=4$, Brugada syndrome $n=2$, other $n=17$ ). (See Fig. 1.)

Of the 168 patients, 116 had DC ICDs implanted, with the remaining 52 patients receiving an SC device. The patients with SC devices were younger $(12.29 \pm 5.04$ years vs $14.93 \pm$ 4.35 years, $\mathrm{P}=0.0001)$ and smaller $(47.2 \pm 23.8 \mathrm{~kg}$ vs $57.5 \pm$ $21.5 \mathrm{~kg}, \mathrm{P}=0.0025)$ at the time of initial device implantation. The mean follow-up time for patients with DC devices was $3.43 \pm 2.83$ years versus $3.1 \pm 3.33$ years for patients with $\mathrm{SC}$ devices $(\mathrm{P}=0.5)$.

For 78 patients in whom the devices were placed for primary prevention, $42 \%$ received a DC device, whereas $52 \%$ of the 90 patients who had devices placed for secondary prevention received a DC ICD. There was no significant difference in the distribution of device types by primary versus secondary implantation indication $(\mathrm{P}=0.68)$

In the study cohort, 41 patients experienced a total of 139 ADs. Of these, 111 ADs occurred in 29 patients in the DC cohort (24.4\% of the total DC population). The remaining 28 ADs occurred in $12(23.1 \%)$ patients with SC devices. There was no statistically significant difference in the incidence of ADs between patients with single- versus dualchamber devices $(\mathrm{P}=0.788)$.

There were 159 IDs recorded during the same period in 35 patients. Of these IDs, 119 occurred in $28(23.5 \%)$ patients with DC devices and 40 IDs occurred in 7 (13.5\%) patients of the SC cohort. There was no significant difference in the incidence of IDs between patients with single- versus dualchamber devices $(\mathrm{P}=0.12)$.

The study patients were divided into three broad primary diagnostic categories: congenital heart disease (CHD), electrical disorders, and myopathies. We then examined the incidence of patients experiencing at least one ID by diagnostic group. Of patients with a DC device and CHD, 33\% experienced at least one ID versus $37.5 \%$ of patients with an SC device and $\mathrm{CHD}(\mathrm{P}=0.83)$. In the subgroup with electrical disorders, $11 \%$ of the DC group experienced an ID compared to $16 \%$ of those with an SC device $(\mathrm{P}=1.1)$. For the 33 patients with cardiomyopathies and DC device, $32 \%$ experienced an ID, while none of the 18 patients with an SC device had an ID ( $\mathrm{P}=0.004)$. (See Fig. 2.)

Of the patients with a DC device implanted for primary prevention, $26 \%$ experienced an ID compared to $11 \%$ of those with an SC device. For those implanted for secondary prevention, $22 \%$ of patients with a DC device and $16 \%$ of patients with an SC device received IDs. There was no statistically significant difference in the incidence of patients with dual- versus single-chamber devices experiencing IDs when compared by primary or secondary prevention implant indications ( $\mathrm{P}=0.15$ and 0.57 , respectively).

During the study period, no patient had a DC device explanted in favor of an SC device. There were, however, 3 patients who had an SC device exchanged for a DC device. In those 3 patients, 2 of the 3 experienced a total of four IDs.

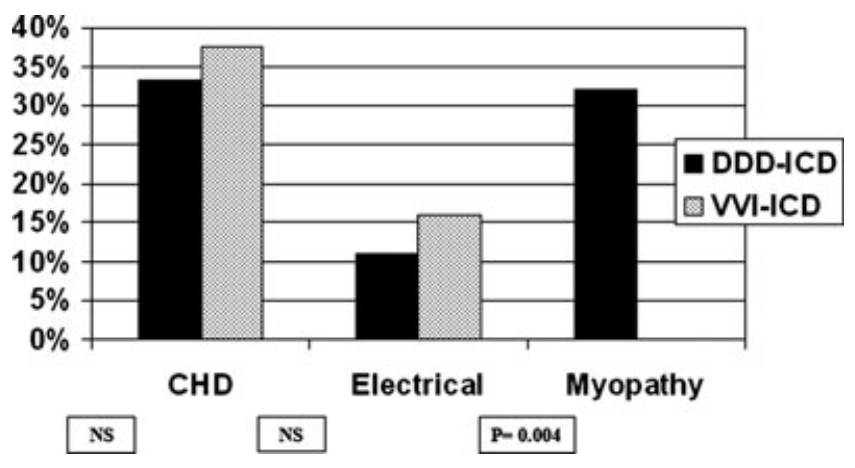

Figure 2. Proportion of patients experiencing inappropriate discharges by primary diagnosis. $C H D=$ palliated congenital heart disease ; Electrical = primary electrical disorder; Myopathy = cardiomyopathy; NS = statistically not significant; $D C=$ dual-chamber ICD; $S C=$ single-chamber ICD. 
However, after the device was "upgraded" to a DC device, all 3 patients experienced IDs (total of 19).

\section{Discussion}

Inappropriate ICD discharges are a common adverse event in patients receiving ICDs. This series was congruent with other series previously published involving pediatric patients with nearly $20 \%$ of all patients experiencing at least one ID. In total, there were more IDs delivered than ADs (159 vs 135). While it is reasonable and perhaps even desirable to sacrifice some specificity for maximal sensitivity when treating potentially life-threatening arrhythmias, all efforts should be made to limit the risk of IDs. The ICD manufacturers have created algorithms to try and discriminate potentially dangerous ventricular arrhythmias from benign tachycardias and biological noise. These have included an analysis of sudden rate changes, rate stability during tachycardia, changes in electrogram morphology, and relationship of atrial and ventricular activity in DDD-ICDs. The ability to include atrial activity in the discrimination of VT from SVT has been thought to be particularly important for young patients, as sinus tachycardia could be misconstrued as VT based purely on rate criteria. The results of this study suggest that the atrial lead does not appear to be beneficial in preventing IDs, and, in fact, patients with a cardiomyopathy and DDD-ICD were more likely to receive an ID than patients with a cardiomyopathy and VVI-ICD. This finding has significant implications for planning device implants in patients in whom an atrial lead may be a burden based on their size and the anticipated need for multiple lead changes during their lifetime.

The delivery of IDs has profound adverse psychological effects. Depression and anxiety in patients simply receiving an ICD is common, ${ }^{12}$ and these sequelae may be accentuated in the aftermath of an ID. ${ }^{13}$ Though adults may tolerate IDs because they accept the device as life saving, ${ }^{14}$ this rationale may be difficult for a child and the family to accept, particularly one whose disease has not yet caused symptoms.

The DDD-ICD, with both atrial and ventricular sensing capabilities, theoretically improves the discrimination of atrial from ventricular tachyarrhythmia. This intuitively should result in a decrease in IDs. The Detect SVT Trial, a multicenter, randomized trial of 400 adults compared the ability of the DDD versus the SC device to appropriately discriminate SVT from VT. ${ }^{8}$ In this study, the likelihood of inappropriate classifications of SVT as VT was significantly decreased with DDD devices. As a result, IDs were reduced. The benefit of the DDD device in the reduction of IDs has been supported by other trials. ${ }^{15,16}$

Other studies have not shown an advantage of DDD devices over VVI devices in the reduction of IDs. ${ }^{9,10}$ Recent addition of and improvement in ventricular electrogram morphology recognition algorithms in SC devices has further improved the ability of VVI ICDs to discriminate between SVT and VT and have been demonstrated to significantly reduce the incidence of IDs. ${ }^{17}$ We found no increased incidence of IDs in patients with SC devices over those with DC devices. Interestingly, there was a trend toward an increased incidence of patients with DC devices experiencing IDs (23.5\% of DDD patients vs $13 \%$ of VVI patients). In this group of young patients, DDD devices did not provide added protection from IDs compared to SC devices.

This study identified young patients with cardiomyopathy and DDD-ICDs to be a group particularly vulnerable to IDs. Of these patients, $32 \%$ experienced at least one ID. In contrast, there were no patients with a cardiomyopathy and VVI-ICD who experienced an ID. Our investigation did not offer clues as to the cause for the increased IDs in the myopathy/DDD-ICD group. While this difference was recognized in the myopathy group, there was no difference in the proportion of patients with electrical disorders and CHD experiencing IDs when classified by device type.

\section{Limitations}

A possible explanation for the difference in IDs could be based upon the device detection algorithm or the tachyarrhythmia detection limits. Due to the large number of devices (within and between device manufacturers) implanted over the years, we were unable to determine if any specific detection algorithm for either VVI-ICD or DDD-ICD device was more efficacious. The implanted devices were primarily manufactured by the Guidant corporation (Natick, MA, USA) (VVI-ICD $=20$, DDD-ICD $=54$ ) or Medtronic (Minneapolis, MN, USA) (VVI-ICD $=31$, DDD-ICD $=62)$, with one St. Jude device (St. Paul, MN, USA). The devices were equally divided among the different diagnoses, which should remove any bias regarding specific devices being more prone to IDs in a particular diagnostic category. Of 93 patients with Medtronic devices, 17 experienced IDs, while 18 of 74 patients with Guidant devices had IDs $(\mathrm{P}=0.34)$. It is also possible that the primary cardiologists presumed that DC devices were better at discriminating SVT from VT, and therefore programmed the tachycardia detection threshold to a lower rate for DDD-ICD devices (conversely programmed the tachyarrhythmia detection rate higher for VVI-ICD devices), increasing the probability of an ID in the DDD-ICD group. However, most of the devices were programmed by pediatric electrophysiologists who tend to set VT detection rates at 220 beats/minute (bpm). The programmed detection rates were not included in this analysis, and this may be an area of future investigation. Additionally, data regarding the pacing mode and whether the atrial lead were used for sensing was not included in the data collection. As this study was retrospective in nature, not all of the raw tracings were available for review. In many patients, the appropriate versus inappropriate distinction was made by searching clinic notes

TABLE 1

Etiology of Inappropriate Discharges

\begin{tabular}{lcc}
\hline & Single Chamber & Dual Chamber \\
\hline No. of patients with ID & 7 & 28 \\
No. of patients ID cause known & 3 & 11 \\
Causes of ID & No. of patients (No. of IDs) \\
Lead fracture & $1(4)$ & $1(6)$ \\
Lead noise & $1(1)$ & $1(6)$ \\
Atrial tachycardia & $1(1)$ & $5(18)$ \\
Sinus tachycardia & 0 & $2(2)$ \\
Device malfunction & 0 & $1(1)$ \\
T-wave oversensing & 0 & $1(1)$ \\
\hline
\end{tabular}


from the primary electrophysiologist. When the etiology of the ID was available, it was tabulated and is presented in Table 1.

\section{Conclusion}

In this nonrandomized, retrospective study of young patients with ICDs implanted for both primary and secondary prevention, DDD-ICDs did not provide additional protection from IDs compared to VVI-ICDs. Continued improvements in detection algorithms are likely to further reduce the incidence of IDs occurring with SC devices. Based on these results, a significant consideration should be given to using a VVI-ICD rather than a DDD-ICD if there is no indication for bradycardia pacing.

\section{References}

1. The Antiarrhythmics versus Implantable Defibrillators (AVID) Investigators: A comparison of antiarrhythmic drug therapy with ICD in patients resuscitated from near fatal ventricular arrhythmia. N Engl J Med 1997;337:1576-1583.

2. Connolly SJ, Gent M, Roberts RS, Dorian P, Roy D, Sheldon RS, Mithchell LB, Green MS, Klein GJ, Obrien B: A randomized trial of the implantable cardioverter defibrillator against amiodarone. Circulation 2000;101:1297-1302.

3. Moss AJ, Hall WJ, Cannon DS, Daubert JP, Higgins SC, Klein H, Levine JH, Saksena S, Waldo A, Wilbur D, Brown MW, Heo H: Improved survival with an ICD in patients with coranary disease at high risk for ventricular arrhythmia: Multicenter automatic defibrillator implantation trial investigators. N Engl J Med 1996;335:19331940.

4. Korte T, Koditz H, Niehaus M, Paul T, Tebbenjohanns J: High incidence of inappropriate ICD therapies in children and adolescents with implantable cardioverter defibrillator. Pacing Clin Electrophysiol 2004;27:924-932.

5. Love BA, Barrett KS, Alexander ME, Bevilaqua LM, Epstein MR, Triedman JK, Walsh EP, Berul CI: Supraventricular arrhythmias in children and young adults with implantable cardioverter defibrillators. J Cardiovasc Electrophysiol 2001;12:1097-1101.

6. Schron EB, Exner DV, Yao Q, Jenkins LS, Steinberg JS, Cook JR, Kutalek SP, Friedman PL, Bubien RS, Page RL, Powell J, for the AVID
Investigators: Quality of life in the antiarrhythmics versus implantable defibrillators trial: Impact of therapy and influence of adverse symptoms and defibrillator shocks. Circulation 2002;105:589-594.

7. Veltmann C, Borggrefe M, Schimpf R, Wolpert C: Fatal inappropriate ICD shock. J Cardiovasc Electrophysiol 2007;18:326328.

8. Friedman PA, McClelland RC, Bamlet WR, Acoste H, Kessler D, Munger TM, Kavesh NG, Wood M, Daoud E, Massumi A, Schuger C, Shorofsky S, Wilkoff B, Glikson M: Dual-chamber versus singlechamber detection enhancements for implantable defibrillator rhythm diagnosis. Circulation 2006;113:2871-2879.

9. Deisenhofer I, Kolb C, Ndrepepa G, Schreieck J, Karch M, Schmieder S, Zrenner B, Schmitt C: Do current dual chamber cardioverter defibrillators have advantages over conventional single chamber cardioverter defibrillators in reducing inappropriate therapies? A randomized, prospective study. J Cardiovasc Electrophysiol 2001;12:134-142.

10. Theuns DA, Klootwijk AP, Goedhart DM, Jordaens LJ: Prevention of inappropriate therapy in implantable cardioverter-defibrillators: Results of a prospective, randomized study of tachyarrhythmia detection algorithms. J Am Coll Cardiol 2004;44:2362-2367.

11. Eberhardt F, Bode F, Bonnemeier H, Boguschewski F, Schlei M, Peters W, Wiegand UK: Long term complications in single and dual chamber pacing are influenced by surgical experience and patient morbidity. Heart 2005;91:500-506.

12. Sola CL, Bostwick JM: Implantable cardioverter defibrillator, induced anxiety and quality of life. Mayo Clin Proc 2005;80:232-237.

13. Kamphuis HC, deLeeuw JR, Derksen R, Hauer RN, Winnubst JA: Implantable cardioverter defibrillator recipients: Quality of life in recipients with and without ICD shock delivery: Q prospective study. Europace 2003;5:381-389.

14. Ahmad M, Bloomstein L, Roelke M, Berstein AD, Parsonnet V: Patient's attitudes toward implanted defibrillator shocks. Pacing Clin Electropysiol 2000;23:934-938.

15. Fan K, Lee K, Lau CP: Dual chamber ICD benefits and limitations. J Interv Electrophysiol 1999;3:239-245.

16. Sticherling C, Schaumann A, Klingenheben T, Hohnloser SH: First worldwide clinical experience with a new dual chamber implantable cardioverter defibrillator. Advantages and complications. Europace 1999;1:96-102.

17. Klein GJ, Gillberg JM, Tang A, Inbar S, Sharma A, UnterbergBuchwald C, Dorian P, Moore H, Duru F, Rooney E, Becker D, Schaaf K, Benditt D, for the Worldwide Wave Investigators: Improving SVT discrimination in single-chamber ICDs: A new electrogram morphology-based algorithm. J Cardiovasc Electrophysiol 2006;17:1310-1319. 